\title{
Different Structures of PVA Nanofibrous Membrane for Sound Absorption Application
}

\author{
Jana Mohrova ${ }^{1}$ and Klara Kalinova ${ }^{2}$ \\ ${ }^{1}$ Department of Nonwovens, Faculty of Textile Engineering, Technical University of Liberec, Studentska 2, \\ 46117 Liberec, Czech Republic \\ ${ }^{2}$ Centre for Nanomaterials, Advanced Technologies and Innovations, Technical University of Liberec, Studentska 2, \\ 46117 Liberec, Czech Republic \\ Correspondence should be addressed to Klara Kalinova, klara.kalinova@tul.cz
}

Received 12 December 2011; Revised 13 February 2012; Accepted 16 February 2012

Academic Editor: Tong Lin

Copyright (๑) 2012 J. Mohrova and K. Kalinova. This is an open access article distributed under the Creative Commons Attribution License, which permits unrestricted use, distribution, and reproduction in any medium, provided the original work is properly cited.

The thin nanofibrous layer has different properties in the field of sound absorption in comparison with porous fibrous material which works on a principle of friction of air particles in contact with walls of pores. In case of the thin nanofibrous layer, which represents a sound absorber here, the energy of sonic waves is absorbed by the principle of membrane resonance. The structure of the membrane can play an important role in the process of converting the sonic energy to a different energy type. The vibration system acts differently depending on the presence of smooth fibers in the structure, amount of partly merged fibers, or structure of polymer foil as extreme. Polyvinyl alcohol (PVA) was used as a polymer because of its good water solubility. It is possible to influence the structure of nanofibrous layer during the production process thanks to this property of polyvinyl alcohol.

\section{Introduction}

The porous fibrous materials, usually used for application in the sound absorption field, absorb sonic waves especially at higher frequencies. The work of Kalinova and Veverka [1] is concerned with topic of absorption the sonic waves by nonwoven materials at lower sound frequencies. The paper deals with a possibility of combining the effect of porous fibrous material and the effect of nanofibrous material which absorbs sound due to the vibration of the membrane. At the moment the sonic waves are in contact with the surface of thin nanofibrous membrane, there is the movement of membrane. The sound energy is converted to kinetic energy and partly to thermal energy. The nanofibers produced by electrospinning process have a large specific surface area [2]. Thanks to the large specific surface of fibers as well as small pores size, the nanofibrous layers have different properties in comparison with currently produced porous fibrous material.

The process parameters of electrospinning are important to the morphology of nanofibers [2]. The work of De Vrieze at al. [3] describes the impact of temperature and relative humidity (RH) on fiber diameter. The fineness of fibers is possible to be affected by RH depending on the chemical nature of the polymer. The collection distance can be used to control the fibers diameter as well. For example, paper [4] describes the case of the decreasing diameter of nanofibers with increasing collection distance by using of PVA solution. With reference to the article [5], the influence of the tiptarget distance to morphology of fibers is not critical. This work shows resulting values of average fiber diameter by application of various electrical potential in electrospinning process too.

Works [4, 6-8] describe effect of polymer solution concentration and its influence on nanofiber diameter by process electrospinning. The average diameter of nanofibers increases nonlinearly with increasing concentration of the solution. The interfiber spacing increases along with this [7]. Article [8] describes a correlation between molecular weight, concentration of solution and average fibers diameter. Type of polymer solution influences the morphology of electrospun fibers. The work [5] deals with an influence of solvent 


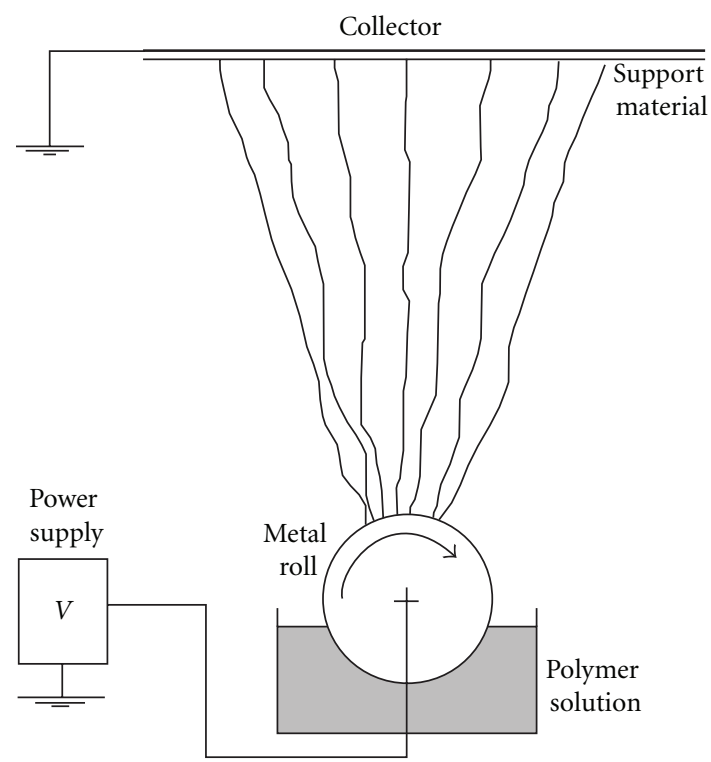

Figure 1: Production of nanofibers layer by electrospinning process.

used for polymer solution to the resultant fibers average diameters in nanofibers layer. This article shows the results of nanofiber parameters by using PVA dissolved in acetic acid and in second case solely aqueous solution of polyvinyl alcohol. In the first case the values of diameters were two times smaller in comparison with the aqueous solution.

The structure of thin nanofibrous membrane can vary as noted above, and therefore the sound absorption properties differ.

\section{Experimental}

2.1. Materials. The water solution of polyvinyl alcohol PVA $\left(\mathrm{M}_{w}=130,000 \mathrm{~g} / \mathrm{mol}\right.$, degree of hydrolysis $\left.88 \%\right)$ Soviol R 16 was purchased from the Novacke Chemicke Zavody, a.s., Slovak Republic, and it was used for preparation of the solution for the experiment. The concentration of prepared PVA solution was $13 \mathrm{wt} \%$. 40\% aqueous solution of Glyoxal (7.5 wt \%) and 85\% phosphoric acid were added as crosslinking agents. The solution containing PVA, distilled water, glyoxal, and phosphoric acid was vigorously stirred at room temperature.

2.2. Production of Thin Nanofibrous Membranes. Technology of electrospinning was used for production of thin nanofibrous layers. The method of using a roll to carry out the polymer solution to collector was described in the patent [9]. Figure 1 indicates the production of electrospun fibers from the surface of the metal roll. The nanofibers are collected by support material. The distance between the surface of the roll and the collector was $10 \mathrm{~cm}$. Voltage of $50 \mathrm{kV}$, relative humidity of $\mathrm{RH} 30 \%$, and temperature of $22^{\circ} \mathrm{C}$ were applied during the course of electrospinning.

The final layer is crosslinked by hot air at temperature of $140^{\circ} \mathrm{C}$ for $7 \mathrm{~min}$. The nanolayer (without support material) is placed to a supporting frame. The structure and sound absorption have been evaluated. Then water vapour or liquid water was applied to these thin membranes. Difference between thin nanofiber membrane (Figure 2(b)) and thin polymeric foil (Figure 2(a)) is markedly observable by macroscopic analysis.

Series of attempts preceded the experiment with using of water vapour for change of structure of PVA nanofiber layer. There were used water vapour because the PVA is water soluble, and this gives possibility to change of membrane structure. Advantage is that the thin nanofiber layers were locked in frames (Figure 2) before application of water vapour. This gives us the opportunity to measure the values of sound absorption coefficient $\alpha[-]$ for nanofiber layers and layers with partly merged fibers when using the same samples. This leads to minimization of potential changes of the other parameters of thin membrane (apart from the structure).

Water vapour was applied to the surface of nanolayer (for 10 to 120 seconds) in order to change the structure of membranes containing nanofibers (average diameter $280 \pm$ $80 \mathrm{~nm}$ ). One file of samples was located into liquid (water) for 60 second. The mass area of PVA layer can be changed depending on the amount of glyoxal in polymer solution and time of water action. The fall of mass of PVA nanofiber layer depending on water action time is decreasing with increasing percent of glyoxal in PVA solution [2]. At this case higher weight percentage of glyoxal and short time of water action resulted in merged fibers to polymeric foil without significant weight loss.

2.3. Characterization. The scanning electron microscope (SEM) Desktop Phenom with BSE detector working at an acceleration voltage of $5 \mathrm{kV}$ was used for characterization of morphology of nanofiber layers. The values of fiber diameters were obtained by image analyzer Lucia G. Calculation of the volume of surface pores [10] is not introduced because a cross-section of nanolayer can deform the inprocess and it can distort the information about thickness of the membrane.

With two-microphone impedance tube Type 4206 with frequency range to $6.4 \mathrm{kHz}$, the values of sound absorption coefficient $\alpha[-]$ were examined. The work [11] deals with a comparison of this method to another method of measurement and describes the absorption coefficient $\alpha$ (value between 0 and 1 ).

\section{Results}

The area weight of all tested thin membranes is $17 \pm 1 \mathrm{~g} / \mathrm{m}^{2}$. Differences between structures of thin membrane are shown in Figure 3. The amount of merged fibers increased with increasing time of water vapour action at the nanofiber layer.

Very important is the question of cross-section of thin membrane in connection with the inner structure of samples. The sample thickness was too small with prevented study of cross-section. If the merged fibers are contained in all membrane thickness, it could not be verified. However, 


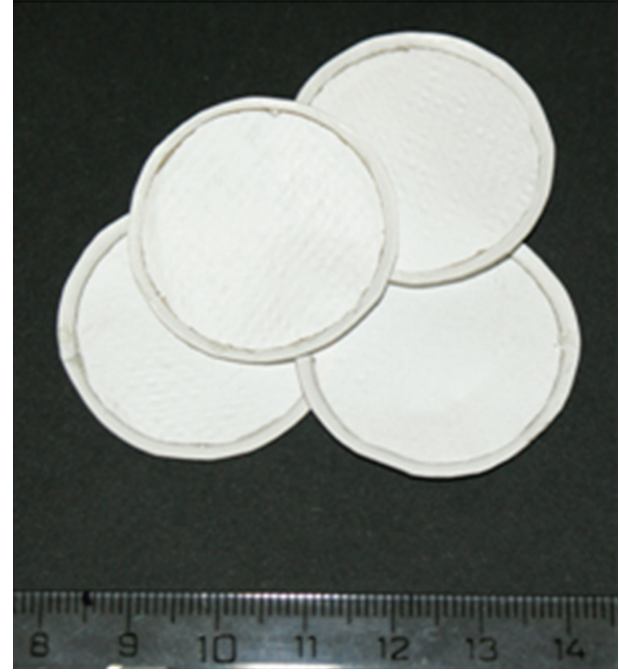

(a)

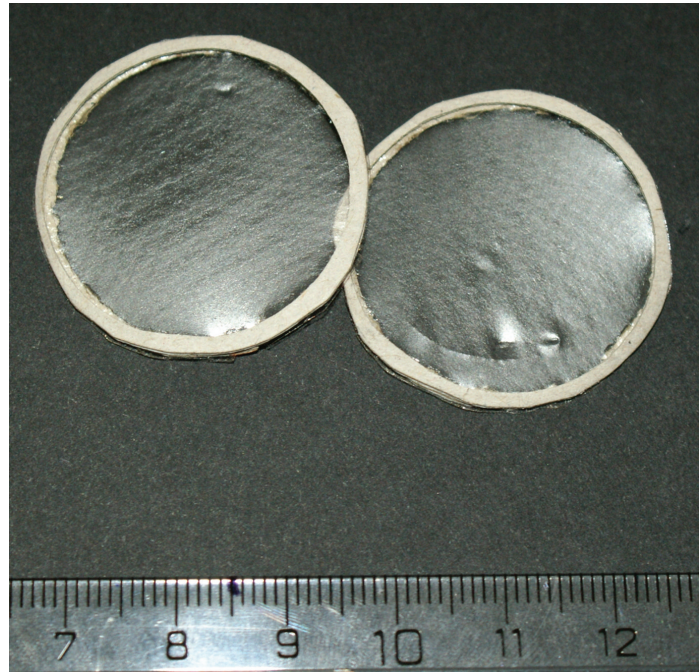

(b)

FIgURE 2: Thin nanofiber membranes (a) and thin polymeric foils (b) supported in frames.
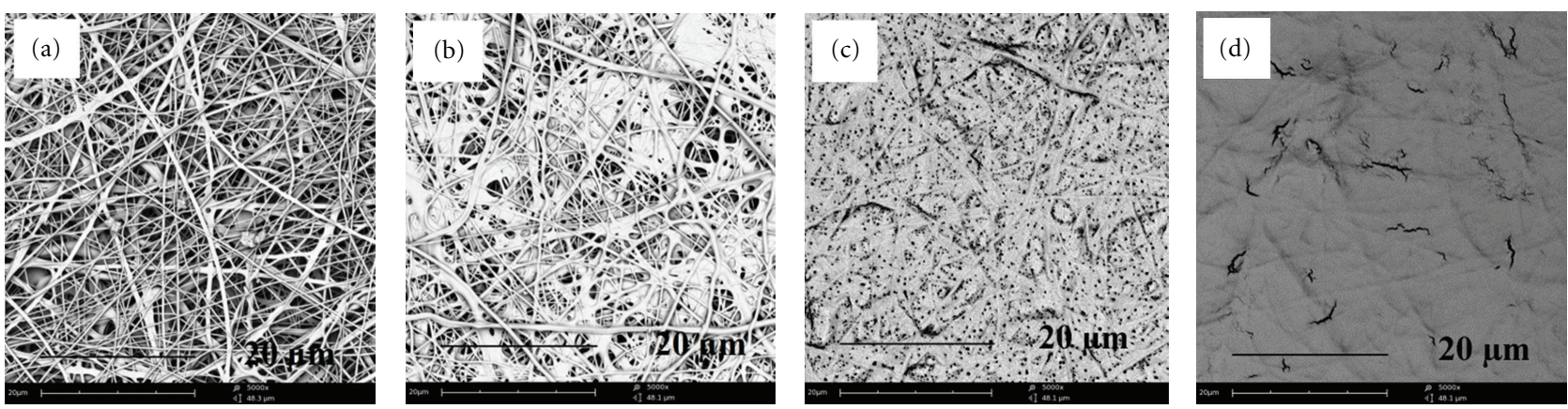

FIGURE 3: Image of surface of PVA nanofiber structures by scanning electron microscope SEM for layers after water vapour action during time of 60 seconds (b) and 120 seconds (c), without liquid water or water vapour action (a) and structure after action of water in the liquid condition to nanofiber layer during the time of 60 seconds (d). Magnification $=5000 \mathrm{X}$ and scale bar $=20 \mu \mathrm{m}$.

method of put samples with frame into two-microphone impedance tube had to be try at range of samples which are not included in this experiment. By this way the impact low thickness of thin membrane and prospective influence to results were reduced as much as possible. Figure 4 pointed to the fact that the shapes of frequency functions are analogical for thin polymeric foil as well as for nanofiber PVA membrane. This result bears witness to the identity of parameters of both membranes (except the structure) as well as the prestressing of the membranes in frame.

The frequency range between $1.5 \mathrm{kHz}$ and $3 \mathrm{kHz}$ is examined because of the area weight of tested thin membranes $\left(17 \pm 1 \mathrm{~g} / \mathrm{m}^{2}\right)$. Resonant frequency of vibrating thin membrane is changed due to its area weight.

Structure of thin membrane after water vapour action contains local area weight irregularity which Figures 3(b) and 3(c) indicate. The thin membrane absorbs sonic waves by resonant principle hence the best at resonate frequency. The mass of thin membrane has influence to the resonate frequency of material. As the time of water vapour action to PVA nanofibers layer is increased, the number of local

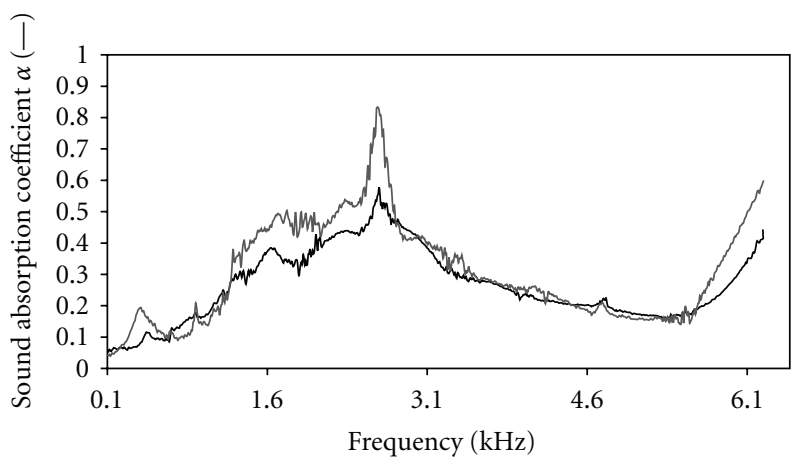

FIgURE 4: Sound absorption coefficient $\alpha[-]$ as function of frequency $[\mathrm{Hz}]$ for thin PVA nanofiber membrane (black line$\alpha \max =0,58)$ and for thin polymer foil (grey line $\alpha \max =0,83$ ).

place with different mass thanks dissolved and merged fibers should increase too. This effect has probably impact to resonant frequency of membrane as unit. Each part of material area resonate at a little bit different frequency 


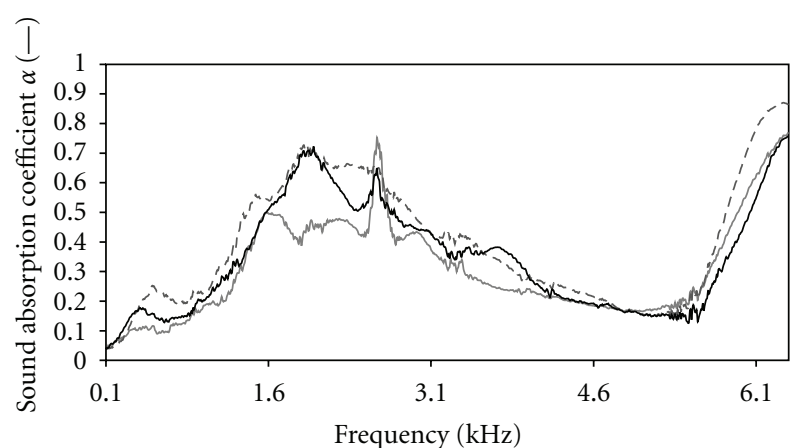

FIgURE 5: Sound absorption coefficient $\alpha[-]$ as function of frequency $[\mathrm{Hz}]$ for PVA nanolayer contains various quantities of merged fibers. The time of water vapour action to nanofibers layers-30 seconds (grey line), 90 seconds (dashed grey line) and 120 seconds (black line).

because of local place and the irregularity of membrane (the above mentioned). This effect has impact on frequency range with good sound absorption which is possible to see in Figure 5. In case of thin polymer film it is possible to suppose lower weight irregularity and the sharp pack of function of frequency (Figure 4).

\section{Conclusions}

The structure of thin membrane can have influence on the amount of absorbed acoustic energy. The possibility of a movement of nanofibers in structure and inner friction in polymer layer has an impact on the final absorption properties of the material. Results achieved for confrontation of polymeric foil and nanofiber membrane differed from the preceding research [12] probably because of the question of material rigidity.

With increasing irregularity in area of material surface, the absorbed frequency range is increased. But there is a limit when the irregularity goes down because of merged fiber majority (Figure 3(c)). In this case the absorbed frequency range comes to a peak again (Figure 5(a)).

\section{Acknowledgment}

The contribution was sponsored by the project of the Ministry of Industry and Trade (MPO), FR-TI3/621 "Nanovlákenné kompozitní textilie pro speciální filtrace".

\section{References}

[1] K. Kalinova and J. Veverka, "Resonant effect of nanofibrous sound absorptive material in room acoustics," Building Research Journal, vol. 57, pp. 55-76, 2009.

[2] B. Ding, H.-Y. Kim, S.-C. Lee, D.-R. Lee, and K.-J. Choi, "Preparation and characterization of nanoscaled polyvinyl alcohol fibers via electrospinning," Fibers and Polymers, vol. 3, no. 2, pp. 73-79, 2002.

[3] S. De Vrieze, T. Van Camp, A. Nelvig, B. Hagström, P. Westbroek, and K. De Clerck, "The effect of temperature and humidity on electrospinning," Journal of Materials Science, vol. 44, no. 5, pp. 1357-1362, 2009.

[4] P. Supaphol and S. Chuangchote, "On the electrospinning of poly(vinyl alcohol) nanofiber mats: a revisit," Journal of Applied Polymer Science, vol. 108, no. 2, pp. 969-978, 2008.

[5] U. S. Sajeev, K. A. Anand, D. Menon, and S. Nair, "Control of nanostructures in PVA, PVA/chitosan blends and PCL through electrospinning," Bulletin of Materials Science, vol. 31, no. 3, pp. 343-351, 2008.

[6] T. Lin and X. G. Wang, "Controlling the morphologies of electrospun nanofibres," in Nanofibers and Nanotechnology in Textiles, P. J. Brown and K. Stevens, Eds., pp. 90-110, CRC Press, New York, NY, USA, 1st edition, 2007.

[7] A. Koski, K. Yim, and S. Shivkumar, "Effect of molecular weight on fibrous PVA produced by electrospinning," Materials Letters, vol. 58, no. 3-4, pp. 493-497, 2004.

[8] J. Tao and S. Shivkumar, "Molecular weight dependent structural regimes during the electrospinning of PVA," Materials Letters, vol. 61, no. 11-12, pp. 2325-2328, 2007.

[9] O. Jirsak, F. Sanetrnik, D. Lukas et al., "Method of nanofibres production from a polymer solution using electrostatic spinning and a device for carrying out the method," WO2005024101 (US2006290031), 2006.

[10] D.-M. Wang, T.-T. Wu, F.-C. Lin, J.-Y. Hou, and J. Y. Lai, "A novel method for controlling the surface morphology of polymeric membranes," Journal of Membrane Science, vol. 169, no. 1, pp. 39-51, 2000.

[11] J. C. Le Roux, J.-P. Dalmont, and B. Gazengel, "A new impedance tube for large frequency band measurement of absorbing materials," in Proceedings of the American Institute of Physics (Acoustic'08), Paris, France, June 2008.

[12] K. Kalinova, Nanofibrous Material as a High-Efficient Sound Absorbent, NANO FOR THE 3RD MILLENNIUM-NANO FOR LIFE, Praque, Prague, Czech Republic, 2007. 

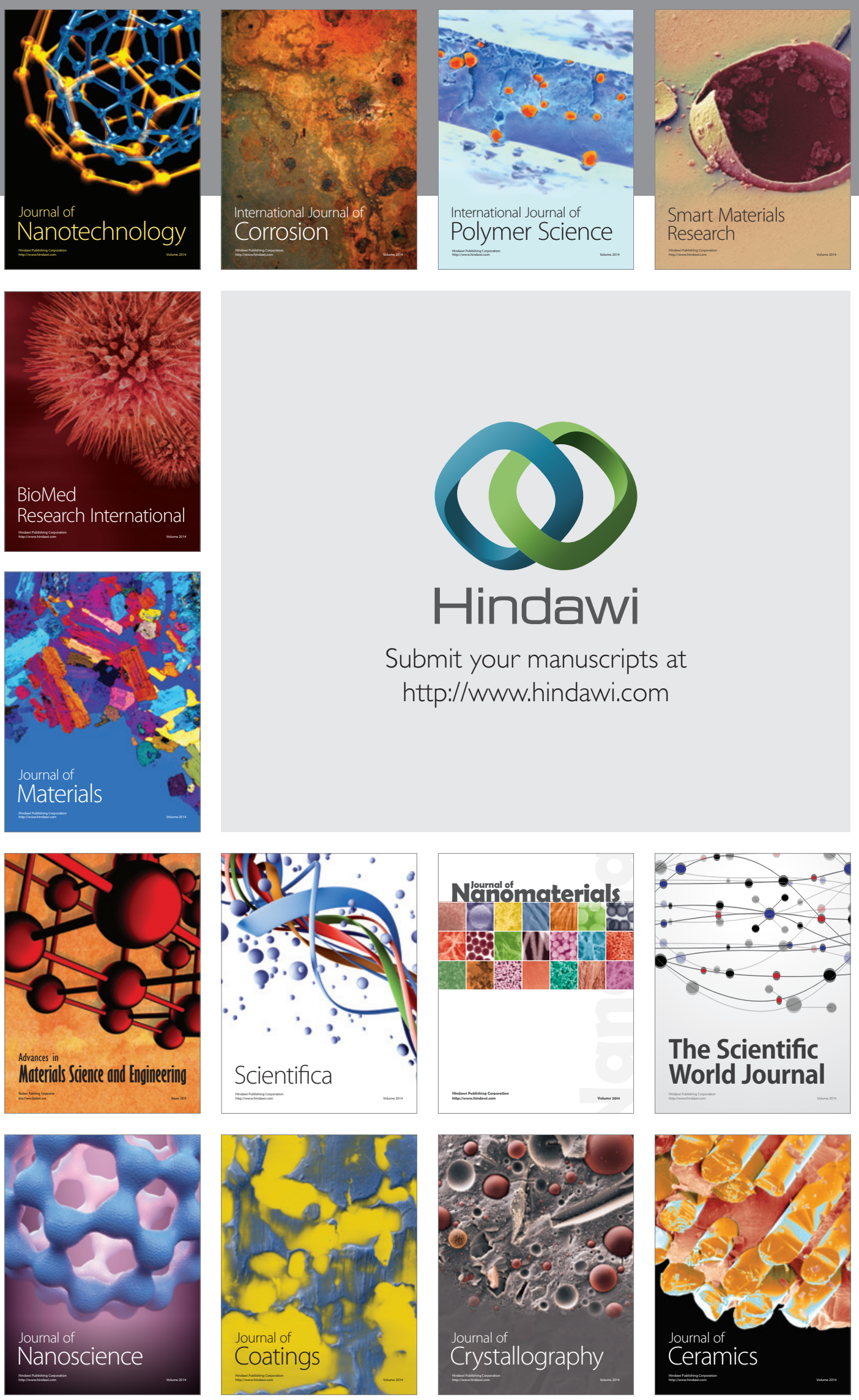

The Scientific World Journal

Submit your manuscripts at

http://www.hindawi.com

\section{World Journal}

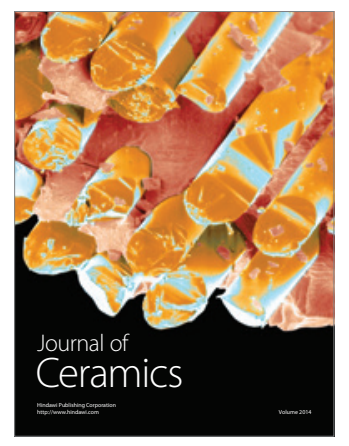

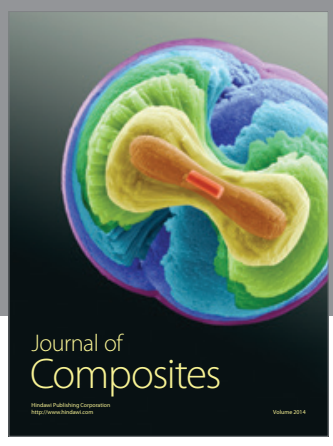
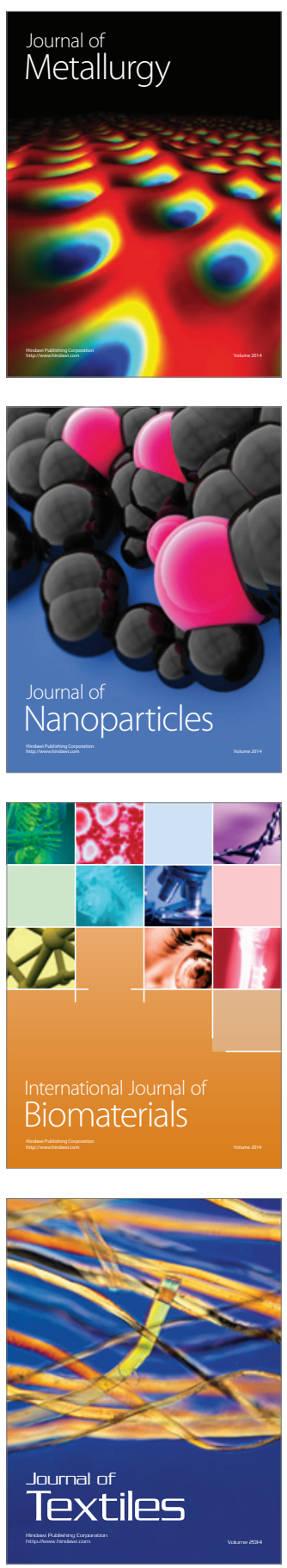